Website: http://revistas.lamolina.edu.pe/index.php/acu/index

(C) Universidad Nacional Agraria La Molina, Lima - Perú

\title{
Diagnóstico del sector agroalimentario de Ecuador: una aproximación al ecosistema agroemprendedor
}

\author{
The food industry in Ecuador: approach to their entrepreneurial ecosystems
}

\author{
Javier Burbano Salazar $^{1 *}$ \& Ma. Ángeles Fernández Zamudio ${ }^{2}$ \\ * Autor de correspondencia
}

\section{Resumen}

En el presente trabajo se revisan los principales rasgos del sector agroalimentario en Ecuador, a la vez que se definen las características principales de los ecosistemas emprendedores. Se repasan, los aspectos sociales y económicos que más influyen en este sector, con el fin de identificar los que deberían mejorarse en pro de incrementar el emprendimiento en el mismo. Para llevar a cabo la investigación se ha procesado información bibliográfica y datos estadísticos de instituciones gubernamentales, además se ha contado con la colaboración de diferentes agentes que participan en la cadena de valor agroalimentaria en Ecuador, a los que se ha consultado para conocer su percepción y valoración sobre las cuestiones centrales del trabajo. Como síntesis de los principales resultados, se han elaborado las matrices DAFO y CAME en las que se recopilan las limitaciones y también potencialidades a ser explotadas para impulsar un emprendimiento más tecnificado en el sector agrario. El trabajo se finaliza con una serie de propuestas que se consideran prioritarias y deberían ser implementadas con este fin.

Palabras clave: Sector agroalimentario; emprendimiento agrario; medio rural; ecosistema emprendedor; Ecuador.

\begin{abstract}
This paper reviews the main features of the agri-food industry in Ecuador, while defining the main characteristics of entrepreneurial ecosystems. They review the social and economic aspects that most influence this sector, to identify those that should be improved to increase entrepreneurship in it. To carry out the research, bibliographic information and statistical data of governmental institutions have been processed, in addition to the collaboration of different agents that participate in the agri-food value chain in Ecuador, who have been consulted to know their perception and assessment on the central issues of work. As a summary of the main results, the SWOT and CAME matrices have been drawn up in which the limitations and potentials to be exploited are gathered to promote a more technically advanced enterprise in the agricultural sector. The work is finalized with a series of proposals that are considered priority and should be implemented for this purpose.
\end{abstract}

Keywords: Agricultural sector; agricultural entrepreneurship; rural environment; entrepreneurial ecosystem; Ecuador.

\section{Introducción}

La agricultura desde siempre ha sido uno de los principales componentes de la economía ecuatoriana, generadora de empleo y por consiguiente de ingresos a las personas y familias que hacen de esta actividad su medio de vida.

A pesar de ello la historia de la agricultura ecuatoriana da cuenta de tres reformas agrarias relevantes, Jordán (2003), indica que la primera reforma de 1964 estuvo marcada por el quehacer heredado de la época colonial, de manera que en pocas superficies se concentraba el mayor número de explotaciones. La convulsión social de aquel entonces provocada por los golpes militares y la no solución de los problemas de distribución de tierras, causó que en 1973 se aprobara una segunda Ley de Reforma Agraria, profundizando los problemas de minifundios, pues muchos de los terratenientes vendían las tierras que se consideraban más productivas, según Martínez Valle (2007), una muestra de la inequidad en la distribución se constata a través del índice de Gini el cual en el período de 1974 a 2001 apenas pasó de 0,85 a 0,80.

En 1979 el Ecuador vuelve a la democracia, envuelto en guerras con los países vecinos y por grandes casos de corrupción, incentivados por los beneficios que obtenían los políticos del "Boom Petrolero". Además, se registraron numerosas manifestaciones internas ocasionadas por el deterioro de la vida rural, reabriéndose la posibilidad de 
una nueva modificación de la ley agraria, concretándose en la Ley de Desarrollo Agrario de 1994. Para López (1994) la finalidad de esta modificación era, entre otras cosas, evitar que se promulguen nuevas reformas, independizar a las instituciones públicas del gobierno en la resolución de conflictos y facilitar la inserción en el comercio global eliminando las barreras comerciales. En la actualidad el Ecuador cuenta con la Ley de Fomento y Desarrollo Agrario, dejando atrás las viejas reformas.

A pesar de los vaivenes en la legislación agraria, este sector ha sido por décadas uno de los pilares principales de la economía del país. Sin embargo, en los últimos años su grado de participación en el PIB ha perdido peso; a pesar de ello el PIB agropecuario que forma parte del PIB Total industrial, ha mostrado un crecimiento, evidenciando la importancia de este para la composición del PIB Total Industrial, pues en el período 2003 - 2015, ha pasado de alrededor de 3,1 a 7,6 millones de dólares.

Así mismo el sector agrícola del Ecuador, es el que más empleo genera. Del total de la población económicamente activa a nivel rural, el $97 \%$ se encontraba empleada en 2014, según los datos de la Encuesta Nacional de Empleo, Desempleo y Subempleo, recogida por el Instituto Nacional de Estadísticas y Censos (INEC). En los datos de la encuesta se puede observar el fuerte vínculo que tiene Ecuador con lo agrario, y la gran dependencia de la agricultura que tiene la población rural, la cual se dedica en un 58,5\% a actividades de extracción de recursos de la naturaleza, es decir, al sector primario (Figura 1).

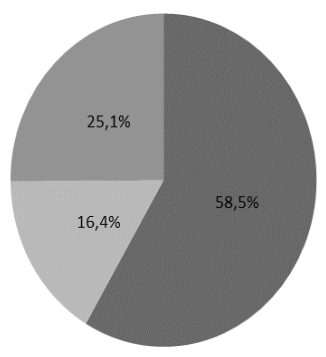

Primario - Secundario - Terciario

Figura 1. PEA rural según el sector económico

Nota: Elaboración propia a partir de los datos de la Encuesta Nacional de Empleo, Desempleo y Subempleo 2014 recogidos por el INEC

Por su parte la Figura 2, muestra la distribución por rama de la actividad, distinguiéndose para ello si se trata de población rural o urbana. Se observa que el 57,7 \% de la Población Económicamente Activa Rural (PEA rural), se dedica directamente a actividades de agricultura, ganadería, acuicultura y pesca, frente al 8,2 \% que lo haría la población urbana. Datos que permiten entender la importancia del sector para las familias del medio rural y que hacen de esta su forma de vida.

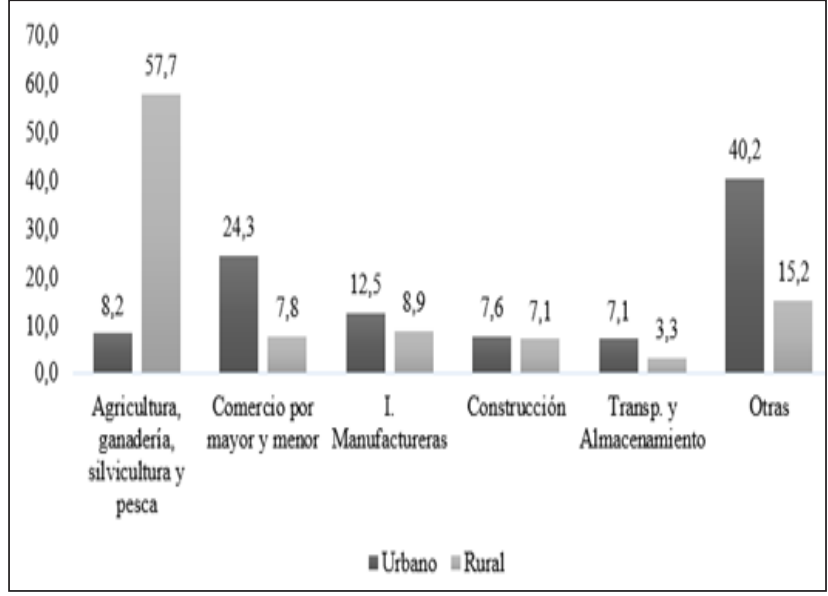

Figura 2. Distribución de la PEA según la rama de actividad Nota: Elaboración propia a partir de los datos de la Encuesta Nacional de Empleo, Desempleo y Subempleo 2014 recogidos por el INEC

Otro dato que muestra la evolución del sector, es el crecimiento de las Unidades de Producción Agrarias (UPA's), que según datos del III Censo de Agropecuario del año 2000 y recogidos por el (INEC), en el Ecuador existen un total de 842882 UPAs que agrupan un área de cultivo de 12355,830 ha. Un crecimiento en más del doble con respecto del primer censo agrario de 1954, en el que las UPA's ascendían a 354234 representando 5 999,700 ha.

A pesar del crecimiento favorable del sector, no se puede considerar que esté desarrollado, entre otras cosas porque aún no se ha logrado que el pequeño y mediano productor agrario a más de extraer también transforme en derivados, dándole mayor valor a su producción. Un caso ejemplificador es el de la cadena productiva del cacao, cultivo de una gran importancia para Ecuador, los productores una vez cosechado venden el mismo en pepas o en baba a los centros de acopio o intermediarios, sin darles mayor valor; estos centros se encargan de venderlo a empresas productoras de chocolates y derivados, así como a empresas para la exportación, y por lo tanto son los que reciben la mayor parte de su valor añadido. Otro indicio de que el sector no ha logrado un desarrollo suficiente se observa en las explotaciones agrarias dedicadas a la agricultura familiar, pues del total de UPA's, registradas en el III Censo Agropecuario, según la FAO (Leporati et al., 2014) el $85 \%$ se dedican a Agricultura Familiar (AF) y solo el $15 \%$ restante puede considerarse una agricultura empresarial. Del porcentaje dedicado a AF, menciona Maleta (2011)pero sin embargo, requiere ser operacionalizada en función de los datos e indicadores disponibles, y ello obliga a pensar con mayor precisión los alcances de la misma. Apuntamos aquí tan solo algunos problemas y la solución que de hecho se le da en el presente contexto. El examen de los datos empíricos sobre las dimensiones y características de la AF primariamente se basa aquí en un reciente estudio de la FAO y el BID que tomó como objeto la agricultura familiar de varios países de la región (FAO-BID 2007, que el $62 \%$ es de subsistencia, el $37 \%$ es de transición y solo un $1 \%$ es una agricultura 
familiar pero consolidada. Se entiende entonces que hay un alto porcentaje de explotaciones cuyo único fin es el de alimentar a sus propietarios, pero son muy pocas aquellas que teniendo estructura familiar tienen opciones de ser viables comercialmente. Parecería obvio, que se deban hacer esfuerzos para intentar que parte de las explotaciones dentro del segmento de transición evolucionen a una tipología de agricultura familiar consolidada. En ese sentido las acciones que fomenten el emprendimiento serían totalmente necesarias para potenciar el valor de todo el sector agroalimentario de Ecuador.

Para avanzar en el desarrollo de emprendimientos agroproductivos en el medio rural, es primordial conocer cómo se encuentra este ahora. Es por lo que, el objetivo del presente estudio es, partiendo de conocer los principales rasgos del sector agroalimentario en Ecuador, detectar los aspectos sociales y económicos que deberían mejorarse en pro de incrementar el emprendimiento en el sector agrícola.

\section{Materiales y métodos}

El diagnóstico del sector agroalimentario ecuatoriano que se realiza en la presente investigación es de tipo descriptivo. Se ha basado en la aplicación de entrevistas a personas conocedoras del sector y que intervienen en este, lo que da paso a la aplicación de técnicas de análisis como las herramientas DAFO y CAME. Se sigue igualmente el método inductivo, pues ha sido preciso inicialmente recopilar numerosa información secundaria, y posteriormente el trabajo con distintos actores del sector agrícola para llegar a las conclusiones que se presentan como parte del trabajo (Dávila, 2006).

Se ha realizado un sondeo exploratorio, consistente en detectar la percepción del sector que tienen diferentes actores, elegidos por ser personas que conocen de primera mano o bien intervienen directamente en el sector agroalimentario. Dado el enfoque del trabajo, centrado en mayor medida en analizar las dificultades que tienen los eslabones productores, el perfil de los consultados ha sido: productores, representantes de asociaciones agrarias y docentes de la rama agraria. El grado de experticia de las personas entrevistadas, permite que, aún con un reducido número de entrevistas la calidad de las respuestas sea suficiente para tener un sondeo prospectivo realista. Las entrevistas fueron personales y extendidas, y las cuestiones planteadas básicamente de naturaleza cualitativa, permitiendo el discurso libre de las personas entrevistadas, con el ánimo de recopilar la máxima información.

Tras recopilar datos oficiales sobre la situación del sector, y en base a las opiniones de las personas entrevistadas, se elaboró tanto la matriz DAFO como la CAME. La DAFO permite hacer un análisis de la situación interna, definiéndose cuales son las Debilidades y Fortalezas del sector agroalimentario en Ecuador, a la vez que la situación externa, lo que queda recogido a través de las Amenazas y Oportunidades que se le atribuyen a este sector en este momento. Para complementar este diagnóstico, se procede después a elaborar una matriz CAME, donde se indican aspectos que pueden ayudar a Corregir las debilidades, a Afrontar las amenazas, Mantener las fortalezas y Explotar las oportunidades (Figura 3).

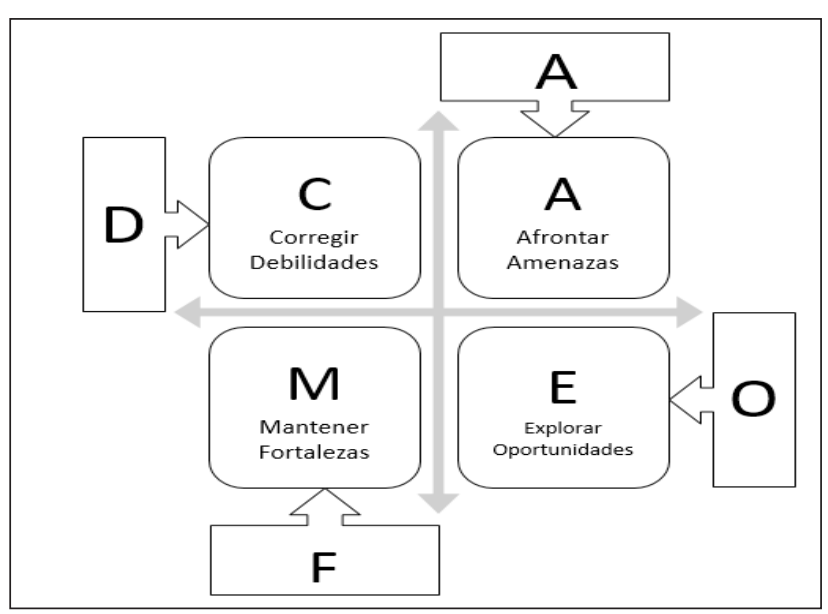

Figura 3. Esquema del diagnóstico que se abarca con las matrices DAFO y CAME

Nota: Elaboración propia a partir de los datos de la Encuesta Nacional de Empleo, Desempleo y Subempleo 2014 recogidos por el INEC.

\section{Resultados y discusión}

Entre los datos claves para entender el sector agroalimentario en Ecuador se señala que, del total de las empresas registradas en el Directorio Empresarial elaborado por el INEC (2014), 10,61 \% se encuentran en el sector de la agricultura, ganadería, silvicultura y pesca. La Tabla 1, muestra las cinco actividades económicas dentro del sector agroalimentario que tienen mayor concentración de empresas. Por otro lado, la Tabla 2 muestra el tamaño de las empresas de acuerdo al sector económico. Sobre el total de empresas registradas, solo el $5 \%$ de las grandes empresas pertenecen al sector de agricultura, ganadería, silvicultura y pesca; mientras que de las microempresas el $11 \%$ corresponde a este sector.

Tabla 1. Empresas por sector económico

\begin{tabular}{lcc}
\hline \multicolumn{1}{c}{ Sector económico / Total } & $\begin{array}{c}\text { Empresas } \\
843,644\end{array}$ & $100 \%$ \\
\hline $\begin{array}{l}\text { Comercio al por mayor y al por menor; } \\
\text { reparación de vehículos automotores y } \\
\text { motocicletas. }\end{array}$ & 308,566 & $36,58 \%$ \\
$\begin{array}{l}\text { Agricultura, ganadería, silvicultura y } \\
\text { pesca. }\end{array}$ & 89,548 & $10,61 \%$ \\
Transporte y almacenamiento. & 87,215 & $10,34 \%$ \\
Industrias manufactureras. & 68,095 & $8,07 \%$ \\
$\begin{array}{l}\text { Actividades de alojamiento y de } \\
\text { servicio de comidas. }\end{array}$ & 63,466 & $7,52 \%$ \\
\hline
\end{tabular}

Nota: Elaboración propia a partir de datos del directorio de empresas y establecimientos 2014, recogidos por el INEC 
Tabla 2. Concentración de empresas por sector económico y tamaño (valores expresados en porcentajes)

\begin{tabular}{|c|c|c|c|c|c|}
\hline Actividad económica & $\begin{array}{c}\text { Micro } \\
\text { empresa }\end{array}$ & $\begin{array}{l}\text { Pequeña } \\
\text { empresa }\end{array}$ & $\begin{array}{c}\text { Mediana } \\
\text { empresa "a" }\end{array}$ & $\begin{array}{c}\text { Mediana } \\
\text { empresa "b" }\end{array}$ & $\begin{array}{l}\text { Grande } \\
\text { empresa }\end{array}$ \\
\hline Agricultura, ganadería, silvicultura y pesca. & 11 & 7 & 9 & 10 & 5 \\
\hline Industrias manufactureras. & 8 & 9 & 9 & 9 & 16 \\
\hline Construcción. & 3 & 6 & 5 & 5 & 4 \\
\hline Comercio al por mayor y al por menor; reparación de vehículos & 36 & 40 & 44 & 45 & 38 \\
\hline Transporte y almacenamiento. & 11 & 6 & 4 & 5 & 3 \\
\hline Actividades de alojamiento y de servicio de comidas. & 8 & 4 & 2 & 2 & 1 \\
\hline Otras actividades de servicios. & 7 & 2 & 1 & 1 & 1 \\
\hline Total & 100 & 100 & 100 & 100 & 100 \\
\hline
\end{tabular}

Nota 1: elaboración propia a partir de datos del directorio de empresas y establecimientos 2014, recogidos por el INEC.

Nota 2: El tamaño de las empresas está dado por el volumen de ventas anuales en millones de dólares americanos y (el número de personas ocupadas): Microempresa < 0,1M\$ (de 1 a 9 personas); Pequeña empresa 0,1-1 M\$ (10-49 personas); Mediana empresa "A" 1-2 M\$ (50-99 personas); Mediana empresa "B" 2-5 M\$ (100-199 personas); Grande empresa >5 M\$ (>200 personas).

Por su parte la posibilidad de emprender en Ecuador y particularmente en el sector agrario, presenta una dualidad. Por un lado, la creación de nuevas empresas, según el Banco Mundial, es cada vez más difícil. El Ecuador con respecto del año 2015, ha bajado tres puestos ubicándose en el $117^{\circ}$ en el ranking de facilidad para hacer negocios de 2016, ya que, en aspectos como la apertura de una empresa, el acceso a créditos, el manejo de permisos de construcción, la obtención de electricidad ha retrocedido entre 4 y 7 puestos, por lo que la puntuación total del Ecuador es de 57,47, quedando por debajo de la media de América Latina y el Caribe (World Bank, 2016).

En contrapartida el Global Entrepreneurship Monitor (2014 y 2015) en su reporte para Ecuador, señala que la Actividad Emprendedora Temprana (TAE) en Ecuador es del 33,6 \% en el año 2015, un punto superior al año anterior donde se situó en 32,6 \% siendo en ambos años la más alta de la región. Así mismo se aprecian crecientes iniciativas de emprendimiento, por ejemplo, la creación de incubadoras y programas de apoyo a emprendedores, al igual que la promoción de fondos de capital semilla y capital riesgo; lo que implica que se involucren en este proceso universidades, ONG's, otras instituciones públicas y empresas privadas. En relación a los fondos concursables como los de capital riesgo, cabe puntualizar que, aún son relativamente escasos y presentan unas condiciones de acceso muy restrictivas, lo que los convierte en una opción de difícil acceso para los agroemprendedores dada la naturaleza de su actividad.

Aun así, hay muchos aspectos que se pueden mejorar. Según Araque (2015), la participación de los actores o promotores del emprendimiento en Ecuador está desarticulada, esto hace que el impulso y el incentivo a emprender se disperse. En cualquier caso, el reporte del GEM (2015), señala que el Ecuador se encuentra por encima de la media de América Latina, en factores que fomentan el clima para el emprendimiento como: educación superior, capacidad para emprender, programas específicos del gobierno, acceso a infraestructura física y normas sociales y culturales. Pero por debajo de la media de la región en otros, como educación y entrenamiento en educación básica, apertura al mercado interno y políticas gubernamentales.
Todas las cuestiones antes mencionadas, a las que se suman las sesiones participativas con los agentes de la cadena agroalimentaria, permitieron elaborar las matrices DAFO y CAME, de las cuales se exponen a continuación los argumentos más relevantes. Mejor que mostrar un diagrama gráfico que recoja de manera simplificada el análisis, se ha optado por detallar un resumen de los principales aspectos que condicionan al sector agrario y a sus empresas. En ellos se concentran los puntos que se creen útiles para incentivar nuevos emprendimientos en el país. Para una mejor comprensión de estos se exponen de forma seguida las Debilidades detectadas junto a los puntos que se tendrían que Corregir para minimizarlas; las Fortalezas y cómo se pueden Mantener; las Amenazas y cómo Afrontarlas, y finalmente las Oportunidades que pueden adoptarse y las vías para Explorarlas.

\section{Debilidades}

- Poca innovación e iniciativas de transformación de la materia prima en producto elaborado.

- En general, sector poco estructurado y cohesionado y predomina la informalidad en las actividades agroproductivas.

- Ausencia de legislación específica que fomente la participación de emprendedores.

- Ausencia de incentivos tributarios a emprendedores e inversionistas.

- $\quad$ Falta de asignaturas de emprendimiento en la docencia de educación primaria, lo que no favorece que haya un impulso emprendedor mucho más global en la población.

- Difícil acceso a tecnologías y crédito para emprendedores.

- Carencia de un programa de incubadoras, que potencien la labor de mentorías y fomenten el ecosistema emprendedor.

- Insuficiente red de sinergias entre agentes del sector, y escaso interés en fomentar el trabajo cooperativo y colaborativo. 


\section{Corregir las Debilidades}

- Fortalecer la legislación nacional en materia de emprendimiento, siendo el papel de la Asamblea Nacional clave para ello.

- Hay que lograr articular la Ley de Educación General Básica, la Ley de Educación Superior, Ley de Régimen Tributario y la Ley de Régimen Laboral con la Ley de emprendimiento, para así potenciar el desarrollo de ecosistemas de emprendimientos agro-productivos con futuro.

- Es preciso estudiar casos asociativos de éxito, que se puedan adaptar y aplicar al sector agrario en Ecuador.

- Se hace necesario promover a través de la Secretaría de Economía Popular y Solidaria el asociativismo y cooperativismo agrario.

- Incrementar los programas formativos en materia de economía agroalimentaria y otras temáticas que favorezcan un sistema productor y transformador más cualificado dentro del sector agroalimentario.

\section{Fortalezas}

- Mano de obra con precios asumibles y que permiten abaratar los procesos productivos.

- Bajos costes en las principales actividades de producción agrícola.

- Existen asignaturas y proyectos que fomentan el emprendimiento en algunas instituciones de Educación Superior.

- Ya hay experiencia de algunas universidades e instituciones públicas en el acompañamiento a emprendedores.

- $\quad$ En el país se dan diferentes ferias y rondas de negocios con buenos resultados, y que son promovidas por universidades e instituciones públicas. Estas podrían ser unos foros de debate donde se conectarán los intereses de todo el sector.

- Existen iniciativas de apoyo a emprendimientos por parte de los Gobiernos Autónomos Descentralizados Provinciales y Municipales.

\section{Mantener las Fortalezas}

- Favorecer el proceso de contratación de mano de la zona, con gratificaciones a los productores que se adhieran a estas iniciativas y permitan una regulación más completa en materia laboral.

- Reconocer a las universidades que trabajen en la formación en emprendimiento y en el apoyo y acompañamiento a emprendimientos agroproductivos, y potenciar actividades que acerquen a los agentes de la cadena de valor a las mismas.

- Normalizar la realización periódica de rondas y ferias de negocios, y crear otros mecanismos para conectar a inversionistas e instituciones financieras con los agroemprendedores.

\section{Amenazas}

- Crisis del petróleo, que durante décadas ha sido clave para el desarrollo económico del país, lo que está teniendo una influencia negativa en otros sectores como el agroalimentario.

- $\quad$ Parte de la normativa vigente llega a afectar de manera negativa, aunque sea indirectamente, a la realización de más actividades de emprendimiento.

- La carga tributaria, por un lado, y los efectos económicos derivados de la globalización de los mercados por otro, no distinguen entre pequeños y grandes productores, lo que supone un agravio sobre los primeros.

- La cadena agroalimentaria no está equilibrada, y el encarecimiento del precio de los insumos afectan especialmente al eslabón productor, mientras que este último apenas participa de los resultados de la comercialización.

\section{Afrontar las Amenazas}

- Estudiar las cadenas de valor de los principales productos agroalimentarios, identificando los cuellos de botellas que causan el encarecimiento de los precios de los productos.

- Articular las distintas leyes de manera que se incentive el emprendimiento tecnificado y la inversión en el sector agrario.

- Implementación de medidas que favorezcan la transparencia y estabilización en materia de precios.

- Sufragar con ciertas ayudas temporales, los insumos más importantes dentro de la producción agrícola, permitiendo un mejor uso a las distintas tipologías de explotaciones, en pro de hacerlas más viables a corto plazo y por lo tanto más competitivas a medio o largo plazo.

\section{Oportunidades}

- Existen algunas líneas de crédito para agricultores, que tienen como fin potenciar la viabilidad de la producción.

- La I+D, aunque sea venida de fuera del país, está llegando poco a poco y es factible acceder a nuevas técnicas de producción y transformación de productos agroalimentarios.

- Sector con amplio potencial para explotar y margen para crecer.

- El cambio de la matriz productiva tradicional podría derivar hacia la adopción de iniciativas con más base tecnológica.

- Desarrollo actual de un Programa de Banco de Ideas e impulso a la implementación de incubadoras con base tecnológica por parte de la Secretaría Nacional de Educación Superior Ciencia y Tecnología. 
- Existen ya experiencia en trabajo colaborativo y cooperativo en otros sectores que podría ser replicado en el sector agrario.

\section{Explorar las Oportunidades}

- Difundir en todas las instituciones de educación superior el Programa de Banco de Ideas y ampliarlo a todo tipo de emprendimientos buscando con ello también las sinergias e interactuaciones con otros ámbitos productivos que necesita el sector agroalimentario.

- Reorientar las líneas de créditos para fomentar el emprendimiento en el sector, haciendo que las ayudas sean más accesibles a los diferentes agentes de la cadena de valor.

- Identificar e implementar aquellos procesos productivos de éxito en otros sectores y países que sean extrapolables a las condiciones del Ecuador.

- Fomentar las agrupaciones de productores y para ello es clave el asociacionismo cooperativo, el cual hay que intentar que esté alineado a un cambio sustancial dentro de la de matriz productiva.

\section{Conclusiones}

Tras el sondeo de la información disponible del sector, y después de haber contado con la opinión de personas intervinientes en la cadena agroalimentaria de Ecuador, se puede concluir que:

Históricamente la agricultura ha sido uno de los componentes principales del PIB del Ecuador, es la principal actividad económica en el medio rural y la que más puestos de trabajo genera. Para las familias del área rural es fundamental el desarrollo de esta, pues el $97 \%$ de la población rural está empleada, y hasta un total del $57 \%$ de dicha población trabaja en la agricultura y ganadería.

Los datos oficiales informan que el $62 \%$ de las explotaciones se dedican al autoconsumo, se evidencia así la importancia que tiene la agricultura en el contexto rural, y explicaría que su carácter sea fundamentalmente familiar y esté centrada principalmente en la producción agrícola primaria. Solo el $37 \%$ de las explotaciones se catalogan como de transición y apenas el $1 \%$ como agricultura familiar consolidada. Esta particularidad también explica que gran parte de las UPAs estén concentradas en pocas extensiones, concretamente el $63 \%$ de las UPA's son menores a 5 ha representando solo el 6,27 \% del total de áreas explotadas.

En el Ecuador existe un marco normativo que propicia la regulación de todas las actividades económicas. Gracias a estos mecanismos institucionales, Ecuador llegó a ocupar en 2014 y 2015 el primer lugar de actividad emprendedora temprana, según el GEM. Sin embargo, sigue siendo necesaria una Ley de Emprendimiento que abarque de manera clara y global, las políticas, normativas, programas y protocolos que regulan la inversión, las formas de financiación, los fondos de riesgo o los beneficios tributarios, todos estos aspectos siguen siendo claves para consolidar los emprendimientos.

La inestabilidad de precios y los vaivenes políticos y en la aplicación de la normativa de generan incertidumbre a lo largo de toda la cadena productiva. A ello se suma la dificultad de acceder a créditos. Contar con un prestamo inicial permitiría disponer del presupuesto necesario para iniciar la actividad, lo que debe hacerse ofreciendo facilidades en su devolución, ya que no hay que olvidar que tras el comienzo de una actividad también es normal que se suceda un cierto tiempo hasta que el negocio empiece a recibir los retornos de dicha inversión.

Precisamente cuando se ha preguntado a las personas consultadas en este estudio cuáles eran los principales problemas del sector agroalimentario señalaron dos: la falta de líneas de créditos, y el acceso a dichos créditos, es decir los excesivos requisitos y trámites burocráticos. En un sector de rentabilidad tan limitada como el agroalimentario sus dificultades se acentúan si no se facilitan los créditos, ya que estos son imprescindibles para adoptar nuevas tecnologías o procesos más tecnificados, o bien simplemente poder sufragar los insumos básicos que exige la explotación, de los que solo ciertos insumos y en puntuales ocasiones son subvencionados.

Se destaca también que la carga impositiva no distingue entre pequeños o grandes productores, por lo que resultaría hasta cierto punto injusta y excesiva para los productores de menor dimensión. Es cierto que hay propuestas interesantes recogidas en el actual Código de la Producción, pero para beneficiarse de las mismas se debe cumplir con unos requisitos mínimos que los pequeños productores no alcanzan.

Todas las cuestiones que se han detallado son los principales argumentos que desmotivan y frenan el lanzamiento de nuevos negocios, y también la innovación en empresas que ya funcionan dentro del sector agroalimentario, y el motivo de que no se aprovechen las grandes bondades del agro y del suelo ecuatoriano.

Finalmente cabe señalar que, aunque la iniciativa privada e individual será clave, hay puntos en los que es crucial la implicación directa de las instituciones políticas. Si la intención es el desarrollo de los ecosistemas de agroemprendimiento, el asociacionismo y la búsqueda de sinergias a través de colaboraciones transversales serán la mejor herramienta para un desarrollo sostenible. Esto es más importante aún en momentos como el actual, en el que miles de familias del medio rural (como por ejemplo de Esmeraldas y Manabí) han sido afectadas por el terremoto de abril de 2016, luchan por reconstruir sus vidas, y deberían tener una opción que les devuelva la dignidad y les permita alcanzar el "Buen Vivir". Este enfoque necesariamente debe incluir la formación de los agentes del sector para que optimicen su participación en las distintas etapas de la cadena agroalimentaria, así como la formación de nuevos profesionales que puedan irse incorporando al sector. La capacitación del personal 
redundará sin duda en un aumento de la competitividad del sector agroalimentario, ya que suele ir aparejada al nivel de innovación de los agronegocios.

\section{Literatura citada}

Araque, W. 2015. Emprendimiento En Ecuador. Ekosnegocios.com, 9 (4), 51. Disponible en: http://doi. org/10.1121/1.4827007

Dávila, N.G. 2006. El razonamiento inductivo y deductivo dentro del proceso investigativo en ciencias experimentales y sociales. Laurus, 1 (12):180-205.

Jordán, F. 2003. Reforma agraria en el Ecuador. Proceso agrario en Bolivia y América Latina, 285-317. Disponible en: http://doi.org/10.1017/ CBO9781107415324.004

Global Entrepreneurship Monitor Consortium. 2014. Global Entrepreneuship Monitor Ecuador 2014. Disponible en: http://www.gemconsortium.org/ report $/ 49224$

Global Entrepreneurship Monitor Consortium. 2015. Global Entrepreneuship Monitor Ecuador 2015. Disponible en: http:/www.gemconsortium.org/ report $/ 49563$

Instituto Nacional de Estadísticas y Censos. 2014. Encuesta Nacional de Empleo, Desempleo y Subempleo. Disponible en: http://aplicaciones3.ecuadorencifras. gob.ec/VDATOS2-war/paginas/administracion/ dashEmpresas.xhtml

Instituto Nacional de Estadísticas y Censos. 2014. Tercer Censo Nacional Agrario 2000. Disponible en: http://www.ecuadorencifras.gob.ec/censo-nacionalagropecuario/

Leporati, M.; Salcedo, S.: Jara, B.; Boero, V. \& Muñoz, M. 2014. La agricultura familiar en cifras. En Agricultura familiar en América Latina y el Caribe. Recomendaciones de Política, 35-56p.

Lopez, E.F. 1994. La ley de desarrollo agrario y la modernizacion. Ecuador Debate.

Maletta, H. 2011. Tendencias y perspectivas de la agricultura familiar en América Latina.

Martínez Valle, L. 2007. Las comunidades rurales pobres y la reforma agraria en el ecuador. En Foro Reforma Agraria, 12p. http://doi.org/10.3406/carav.1980.1501

World Bank. 2016. Doing Business 2016: Measuring Regulatory Quality and Efficiency. Washington, DC: World Bank. DOI: 10.1596/978-1-4648-0667-4. License: Creative Commons Attribution CC BY 3.0 IGO 